\title{
Design of a suspended particles sensor based on laser backscattering theory that used in poultry housing and corral

\author{
Xiande Zhao, Leizi Jiao and Daming Dong*
}

Beijing Research Center of Intelligent Equipment for Agriculture, Beijing Academy of Agriculture and Forestry Sciences, Beijing, China

e-mail: damingdong@hotmail.com

Keywords:component; particle; sensor; backscattering; laser

\begin{abstract}
The suspended particulate matters are one source of the main pollutants in poultry housing and corral. There are also lots of serious pathogens in the air as suspended particles. It is harmful to the health of animals and workers when the density of suspended particulate matter is high. It is significant to detect the amount of particles suspending in the air of poultry housing and corral. In this paper, a kind of sensitive sensor based on the theory of backscattering was designed. The optical and electronic designs are described in detail. After been developed the sensor was tested on its accuracy and stability, and the results were satisfactory.
\end{abstract}

\section{Introduction}

The suspended particulate matters are the particles whose diameters are less than $100 \mu m$.The smaller particles whose diameters are less than $10 \mu m$ (PM10) can get into the internal body through the respiratory system to harm the health and the particles less than $2.5 \mu \mathrm{m}$ (PM2.5) even can increase the mortality risk of patient with lung cancer about 8\% 37\% [1].

Because of the dense breeding and slow exchange of air, a mount of particulate matters suspend in the air as one of the main pollutants [2]. Especially in cold winter, in order to keep the temperature fit to the living of animals, poultry housings and corrals are usually unventilated that lead to the accumulation of the inhalable particles. If workers worked in the environment long time would susceptible to acute or chronic epidemics. Their symptoms would be cough [3], fever, even lung function decline [4]. And the livestock and poultry lived long time in dirty air would cause their immunity reduced, and suffered from various respiratory diseases. Their production efficiency would be serious influenced [5]. The dusts in poultry housing mainly come from feed, bedding, feather, excrements and microorganism. With the characteristics of strong toxicity, high concentration and spread widely [6], the dusts are the main carriers of fungal spores, bacteria, viruses and other organic material.

There are two kinds of methods to detect the suspended particulate matters in air that respectively are sampling methods and non-sampling methods [7]. Sampling methods are based on the air sampling of the measured environment. After filtering and weighing the quality of the obtained particles in the air sample, the particles concentration can be calculated out according to the sampling volume. This kind of methods needs complex operations and long measuring time and it is low degree of automation, and cannot carry out online detection. The non-sampling methods generally include light attenuation method [8], Tapered Element Oscillating Microbalance (TEOM) [9, 10], $\beta$ ray attenuation method [11] and light scattering method [12]. But the light attenuation method is not suitable for the situations that the dust concentration is relatively low and the detection distance is short, because the light attenuation is insensitive in these situations [13]. The accuracy of TEOM method that with complex structure and high operation cost can be reduced in the environment of high humidity. $\beta$-ray attenuation method is an indirect measuring method and the accuracy of measurement is not only concerned with the accuracy of sampling flow rate, but also with the particle composition [14]. The light scattering measurement as a method with high precision, fast speed, wide applicability of particle diameter range, easy to realize remote and real-time detection, is currently used widely $[15,16]$. According to the different angles of light scattering from the air suspended particulate particles, the scattering points out forward scattering and backscattering. The 
present studies about measurement of dust particle concentration based on forward scattering are common [16, 17]. This paper introduces a design of a sensor of air suspended particulate matters based on laser backscattering.

\section{Detection Theories}

The principle of scattering is when light transmitting in inhomogeneous medium and interacting with the medium particles, forcing the particles vibration, and producing radiation of two times. The light scatterings of suspended particles in air depend on the ratio of particle size and the wavelength of incident light. For larger particles, the energy distribution of the scattered light is mainly concentrated in forward. While for smaller particles, the energy distribution of the scattered light is wider. The particle diameter is smaller, and its light scatterings in forward and backward are more uniform. The light scatterings of different particles are shown in Fig.1.

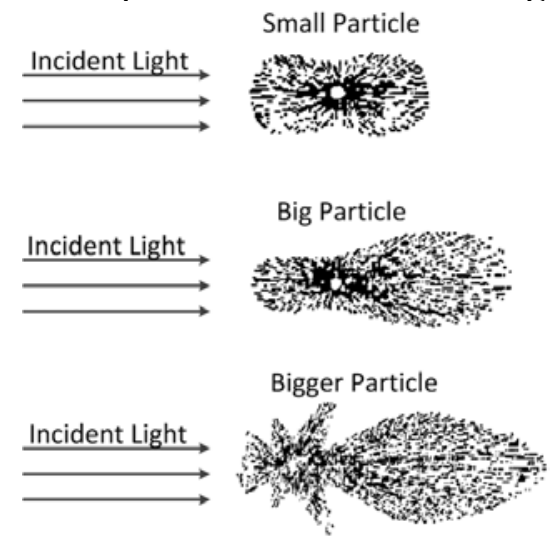

Fig.1 Intensity distribution of light scattering from different scales particles

G.Mie and R.Debye calculated the scattering of electromagnetic waves in detail based on spherical particle models. Based on the Maxwell's equation of electromagnetic field, Mie worked out the strict solution on light scattering, and he concluded the light scattering law of uniform particles with arbitrary components and any diameters [12].

When a beam of parallel light that its wavelength is $\lambda$ and intensity is $I_{0}$, shot on a spherical particle with $\mathrm{D}$ diameter, the scattering intensity $\left(I_{1}\right)$ at the distance of $\mathrm{r}$ can be calculated as the equal (1) shows.

$$
I_{1}=\frac{I_{0}}{r^{2}} N \delta=N \frac{I_{0} \lambda^{z}}{8 \pi^{2} r^{2}}\left[i_{1}(k, m, \theta)+i_{2}(k, m, \theta)\right]
$$

Where:

$N$ is the total number of particles in per unit volume,

$N \delta$ is the scattering coefficient of the unit scatterer,

$\mathrm{K}$ is the dimension parameter,

$m$ is the refractive index of the being measured particles,

$\theta$ is the scattering angle $\left(90^{\circ}<\theta<270^{\circ}\right)$,

$i_{1}(k, m, \theta)$ and $i_{2}(k, m, \theta)$ are the scattering intensity function, respectively represent the parallel and perpendicular scattering intensity of scattering surface.

\section{Sensor Design}

The sensor development mainly includes the optical path design and the electronics design. The optical path design mainly includes the regulation of light source, lens, and detector and the conditioning of their angle and position. The electronics design mainly include light source driver module, scattered light detection module, display module and communication unit. The structure of the sensor is shown in Fig.2. 


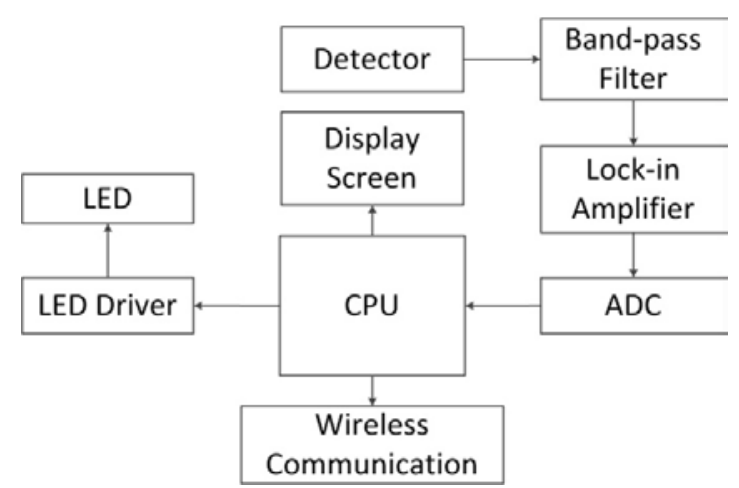

Fig.2 Structure of particle sensor

\section{A. Design of optical structure}

There are many advantages using structure of light backscattering. The most important one is more sensitive and easy to be miniaturized because of the position of light source and detector which are installed in the same side of the sample. In this design, a bright LED at the wavelength of 780nm was chosen as the light source. The angle of divergence was $11^{\circ}$ and the maximum power consumption was about $200 \mathrm{~mW}$. The detector was selected as the S2386 series PIN photodiode (Hamamatsu, Japan). It had a good response at the wavelength of 780nm, and its biggest dark current was at the pA level. The diameter of lens using for converging beam was $2 \mathrm{~cm}$, and the transmittance ratio was as high as $95 \%$ while the focal length is $2 \mathrm{~cm}$.

The light source and photoelectric detector were fixed on a circular circuit board. The detector was welded in the central position of the circuit board, and three LED were arranged at the detector around with equidistant distribution, tilting to the center as a 20 degree angle. the beams of the three LED can be gathered in front of the middle detector, forming a scattering region, which is also the sampling area. The lens was installed in front of the photoelectric detector and the focus was on the sensitive surface of the photoelectric detector that will enhance the gathering ability of scattering light and improve the sensitivity of the sensor. The optical design was shown in Fig.3.

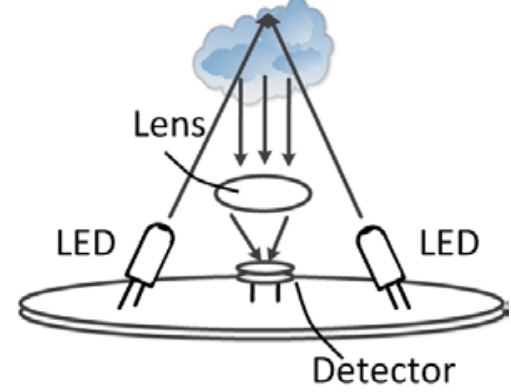

Fig.3 Optical path design

\section{B. Design of electronic system}

The electronic part mainly includes the light source modulation circuit, photoelectric signal conditioning circuit and MCU control system. Because the signal of backscattering is very weak, so the stability of the light source is a high requirement. For eliminating the interference of background noise, the LED driving circuit was designed using $35 \mathrm{KHz}$ sine wave as the driving signal. A sine wave generator (AD9850) was used to generate a sine wave signal, and drive a Darlington sink driver (ULN 2003A). This Darlington driver with high current gain, high working voltage, wide temperature range and strong load capacity, adaptive all kinds of high speed and large power driving system. It was used to provide stable current to the bright LED light in this design. The LED driving current is shown in Fig.4. 


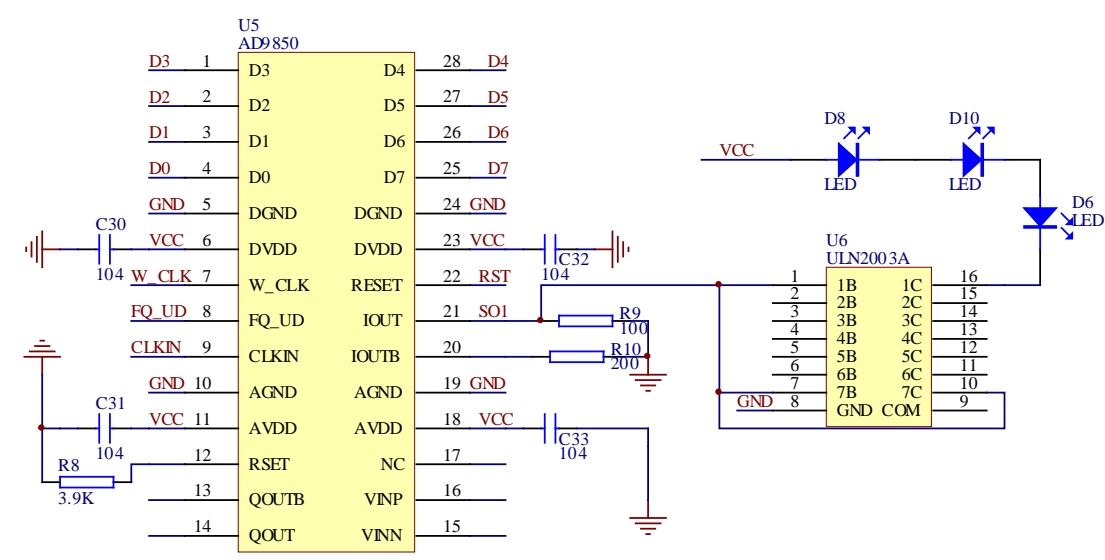

Fig.4 LED Driver circuit

When the light from LED passed through the scattering region, the dust particles on the optical path impacted the photon and scattering occurred. The backscattering light was converged by the lens and focused on the sensitive surface of the photoelectric detector. The detector generated current signal. The signal is weak AC current, had the same frequency (35 KHz) with the light source driving signal. Therefore, the signal conditioning circuit just extracted the signal of $35 \mathrm{KHz}$ and filtered the noise signals of other frequencies, and then, converted the AC signal into DC signal. The signal conditioning circuit is shown in Fig.5.

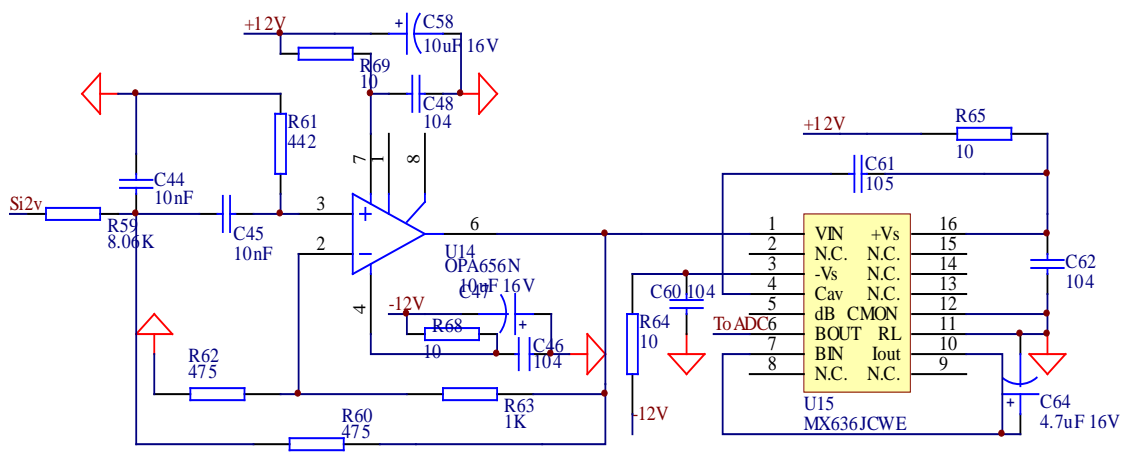

Fig.5 Signal conditioning circuit

The signal was regulated to the stable voltage at $0-2.5 \mathrm{~V}$, then, was acquired by analog to digital converter (ADC). The central processing unit was C8051F007 that integrated with 12 bit ADC and 12 bit DAC. After acquiring the signal, C8051F007 calculated the collected digital signal according to the built-in mathematical model and work out the concentration of the air suspended particulate matters. The result can be outputted in digital form directly through the serial port or converted into analog signal by DAC, and the result can be stored in a built-in 64M flash memory. The sensor's sampling frequency was 15 seconds at a time.

The sensor was calibrated and tested after its design and development. The calibrated method was building a relationship between the voltage value and the concentration value of weight measurement method, and getting the calculation formula between the voltage and the concentration of suspended particulate matters. When the sensor works, it works out the particles concentration base on this formula.

\section{Sensor Test}

The sensor was tested in its accuracy and stability after developed. The accuracy test was carried out by comparing the result with the measured value of one commercialized instrument, Thermo Scientific TEOM1405-DF (America), which based on the method of TEOM. We chose a real chicken farm named Deqingyuan (Yanqing, Beijing,China) as the measurement environment. The measuring position was the middle part of the henhouse and the measuring height was about $1.5 \mathrm{~m}$. The two devices were placed side by side and worked continuously. We recorded the results every hour. The measurement results have been shown in Fig.6. As you can see, the measurement results of the air suspended particulate matters sensor developed independently based on laser 
backscattering were identify with the results of the instrument imported from America based on TEOM, and the error does not exceed 5\%.

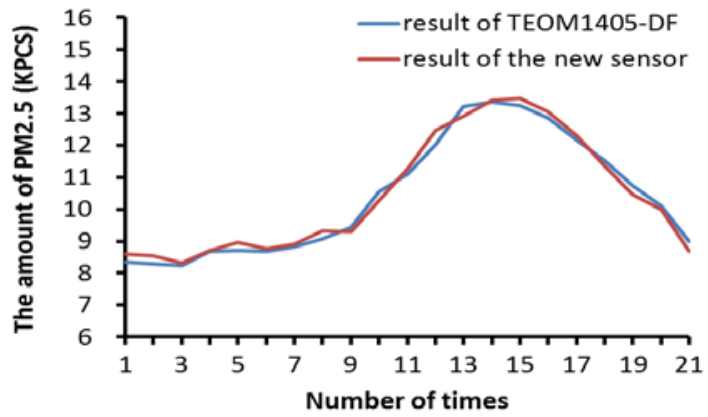

Fig.6 Results of two kind of method

Stability test was executed in a confined space. The sensor was placed in the space and worked for a long time. The space temperature and humidity were kept constant as far as possible. The air circulation rate was slow, and the change of dust concentration was reduced. We recorded the results every hour. In Fig.7, the measurement results are shown as a line. It shows, this sensor has good stability.

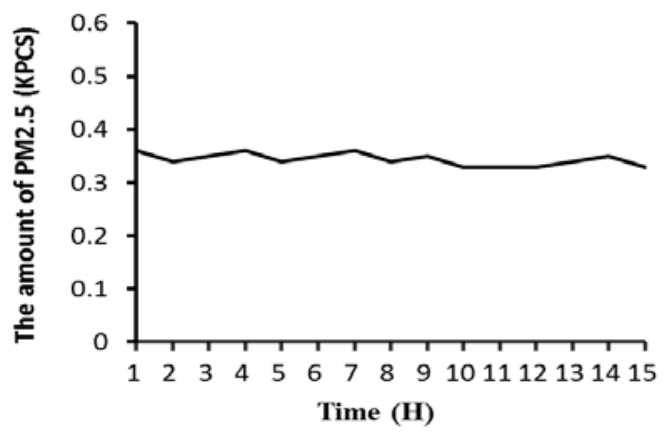

Fig.7 Stable results of the sensor in 15 hour

\section{Conclusion}

An air particulate matters sensor was design based on laser backscattering theory used to apply in poultry housing and corral. It can realize the real-time online measurement and remote data transmission. As the test, it has good accuracy and stability. The sensor designed based on laser backscattering, so it is very sensitive to the smaller diameter particles, and has relatively larger measurement error to the bigger suspended particulate matters. When it used in high humidity environment, the output of the sensor is a bit on the high side.

\section{Acknowledgment}

This work was supported by Beijing Natural Science Foundation (No. 4131002).

\section{References}

[1] J. Lepeule, F. Laden, D. Dockery, and J. Schwartz, "Chronic Exposure to Fine Particles and Mortality: An Extended Follow-up of the Harvard Six Cities Study from 1974 to 2009," ENVIRONMENTAL HEALTH PERSPECTIVES, vol. 120, pp. 965-970, 2012.

[2] F. Liu, W. Gu and X. Lian, "Detection of Dust in Scale Chicken Farm," Animal Husbandry and Feed Science, pp. 9-10, 2012-10-30 2012.

[3] E. Zuskin, J. Mustajbegovic, E. N. Schachter, J. Kern, N. Rienzi, S. Goswami, Z. Marom, and S. Maayani, "Respiratory function in poultry workers and pharmacologic characterization of poultry dust extract," Environ Res, vol. 70, pp. 11-9, 1995-07-01 1995.

[4] K. J. Donham, D. Cumro, S. J. Reynolds, and J. A. Merchant, "Dose-response relationships between occupational aerosol exposures and cross-shift declines of lung function in poultry workers: recommendations for exposure limits," J Occup Environ Med, vol. 42, pp. 260-9, 2000-03-01 2000. 
[5] M. Liu, "Chicks deadly disease of tracheal obstruction," China Poultry, p. 25, 2001-08-15 2001.

[6] J. Cheng, "Experimental study on the dust distribution of closed henhouse in winter,". China Agricultural University, 2006.

[7] T. Li and H. Hu, "Study on henhouse dust detect technique with scatter light," Journal of Chinese Agricultural Mechanization, vol. 35, pp. 186-188, 2014.

[8] L. Xiaohu, "Study on the Calibration Technology of Measuring Dust Concentration by Light Transmission Method," Machinery \&amp; Electronics, pp. 52-54,55, 2014.

[9] J. Ya-ting, G. Jun, X. Wei-min, L. Xin-zhong, and G. Yan-lin, "USING TAPERED ELEMENT OSCILLATING MICROBALANCE METHOD TO DETERMINE THE CONVERSION COEFFICIENT OF ATMOSPHERIC PARTICULATE MATTER TESTED BY LASER DUST MONITOR," Journal of Jinggangshan University (Natural Sciences Edition), pp. 1-6, 2014.

[10] J. Ding, "Research on the stationary pollution source smoke emission concentration monitoring in the oscillating balance method," Resources Economization \&amp; Environment Protection, p. 99, 2014.

[11] L. Zong-lun, Z. Xiu-Liang, P. Li-jing, and L. Li-yan, "Introduce on theory and applications about dust measurement base on $\beta$-ray attendation," CHINA MINING MAGAZINE, vol. 19, pp. 107-109, 2010.

[12] C. Hu, "Investigtion of dust density measurement based on scattering theory,". : Soochow University, 2007.

[13] G. Tian, J. Sun, J. Zhu, and T. Jia, "STUDY ON COMPENSATION OF DUST POLLUTION AND LIGHT INTENSITY DISTRIBUTION IN THE LIGHT SCATTERING DUST SENSOR," JOURNAL OF CHINA COAL SOCIETY, pp. 74-78, 1997-12-25 1997.

[14] Y. Zhang and Y. Zheng, "Comparison of $\beta$-ray Decay Method and Tapered Element Osillating Microbalance Method to Detect PM10," The Administration and Technique of Environmental Monitoring, pp. 21-23, 2002-09-30 2002.

[15] V. P. Dick, "Applicability Limits of Beer' s Law for Dispersion Media with a High Concentration of Particles," Applied Optics, vol. 37, p. 4998, 1998.

[16] Y. Wang, D. Feng and Z. Zhang, "Research on Measuring the Thickness of Soot by Scattered Light," Journal of Shandong Institute of Building Materials, pp. 253-254+258, 2001-09152001.

G. Xu and N. Wang, "Experimental Investigation of the Multiwavelength Forward Light Scattering Flux for Measuring Particle Size Distribution," APPLIED LASER, pp. 61-64, 1997-04-25 1997 